\title{
ON THE IMMERSION PROBLEM FOR REAL PROJECTIVE SPACES
}

\author{
BY I. M. JAMES \\ Communicated by Raoul Bott, November 29, 1962
}

1. Introduction. Let $P^{j}$ denote real projective $j$-space, with its usual differential structure. What is the least dimension of euclidean space in which this manifold can be immersed? The purpose of this note is to prove

Theorem (1.1). If $j=2^{r}-1$ then $P^{i}$ cannot be immersed in $(2 j-q)$ space where

$$
\begin{aligned}
q & =2 r & & (r \equiv 1,2 \bmod 4) \\
& =2 r+1 & & (r \equiv 0 \bmod 4) \\
& =2 r+2 & & (r \equiv 3 \bmod 4) .
\end{aligned}
$$

Hence, and from the Hurwitz-Radon theorem, it follows that if $P^{i}$ can be immersed in $(2 j-k)$-space, when $j+1$ is a power of 2 , then $P^{j}$ admits a $k$-field of linearly independent tangent vectors. Our method is based on the work of Adams [1], Atiyah [2] and Hopf [6]. In [3] Atiyah has proved, by the method of exterior powers, that $P^{i}$ cannot be immersed in $(2 j-p)$-space where $p$ is approximately $j / 2$. When $j+1$ is a power of 2 the value of $p$ in all cases exceeds the value of $q$ in (1.1), and $p / q \sim 2^{r-2} / r$ when $r$ is large.

For $r \leqq 3$ it is known (see Hirsch [5]) that $P^{i}$ can be immersed in $(j+1)$-space. I do not know whether $P^{15}$ can be immersed in 22space, ${ }^{1} P^{31}$ in 53 -space, and so forth.

2. $S$-theory $\bmod p$. Throughout this section we take homology and cohomology with coefficients in the ring of integers $\bmod p$, where $p$ is prime. The $m$-fold suspension of a space $X$ is denoted by $S^{m} X$. A class $\alpha \in H_{r}(X)$ is said to be $S$-spherical if, for sufficiently large $m$, the $m$-fold suspension of $\alpha$ can be represented as the image of the fundamental class on $S^{m+r}$ under the homomorphism induced by a map into $S^{m} X$.

We say that $X$ is mod $p S$-related ${ }^{2}$ to $Y$ if there exists a map of

${ }^{1} \mathrm{Mr}$. B. J. Sanderson has kindly informed me that the existence of this and other immersions is proved in a paper of his to be published in the Proceedings of the London Mathematical Society.

2 We do not need to show that this is an equivalence relation, for present purposes, although it would not be difficult to do so, at least when the spaces are complexes. 
$S^{m} X$ into $S^{n} Y$, for some $m$ and $n$, which induces an isomorphism of $(\bmod p)$ homology groups. We prove

Lemma (2.1). Let $X$ be mod $p$ S-related to $Y$. If a homology class of $Y$ is $S$-spherical then so is the corresponding homology class of $X$.

For let $h: S^{m} X \rightarrow S^{n} Y$ be a map which induces a homology isomorphism. Consider the naturality diagram shown below, where $\xi, \eta$ are Hurewicz homomorphisms and $h_{*}, h_{*}$ are the homomorphisms induced by $h$.

$$
\begin{array}{cc}
\pi_{r}\left(S^{m} X\right) \stackrel{h_{\sharp}}{\longrightarrow} \pi_{r}\left(S^{n} Y\right) \\
\xi \downarrow & \downarrow \eta \\
H_{r}\left(S^{m} X\right) & \underset{h_{*}}{\longrightarrow} H_{r}\left(S^{n} Y\right)
\end{array}
$$

Since $h_{*}$ is an isomorphism it follows that $h_{\sharp}$ is a C-isomorphism, by Theorem 3 in Chapter III of [9], where $\mathcal{e}$ denotes the class of finite abelian groups with order prime to $p$. Hence $\eta h^{*}$ has the same image as $\eta$. Since $h_{*} \xi=\eta h_{*}$ this proves (2.1).

If $X$ is $\bmod p S$-related to a dual of $Y$, in the sense of $S$-theory, we say that $X$ is $\bmod p S$-dual to $Y$. Let $X$ and $Y$ be finite complexes and let $g: X \# Y \rightarrow S^{n}$ be a map, where $X \# Y$ denotes the "smash" product. Let

$$
\phi: H_{n-8}(X) \rightarrow H^{s}(Y)
$$

be the corresponding slant operation ${ }^{3}$ defined with respect to the fundamental class on $S^{n}$. We prove

Lemma (2.2). If $\phi$ is an isomorphism then $X$ is $\bmod p S$-dual to $Y$.

Let $m$ be large in comparison with the dimensions of $X$ and $Y$. Let $Z$ be an $m$-dual of $Y$, in the sense of Spanier [11], with duality map $u: Z \# Y \rightarrow S^{m}$ and associated slant isomorphism

$$
\psi: H_{m-s}(Z) \rightarrow H^{s}(Y)
$$

By (5.8) of [11] there exists a map $h: S^{m} X \rightarrow S^{n} Z$ such that $g^{\prime}$, the $m$-fold suspension of $g$, is homotopic to the composition

$$
S^{m} X \# Y \stackrel{h^{\prime}}{\rightarrow} S^{n} Z \# Y \stackrel{u^{\prime}}{\rightarrow} S^{m+n},
$$

where $h^{\prime}=h \# 1$ and $u^{\prime}$ is the $n$-fold suspension of $u$. It follows from the definition of the slant operation that $\phi^{\prime}= \pm \sigma \psi^{\prime} h_{*}$, as in the dia-

${ }^{3}$ See $\$ 11$ of [10] and [11] generally. We transfer to the reduced groups whenever this operation is under consideration. 
gram below, where $h_{*}$ is induced by $h, \sigma$ is the suspension isomorphism, $\phi^{\prime}$ is the $m$-fold suspension of $\phi$, and $\psi^{\prime}$ is the $n$-fold suspension of $\psi$.

$$
\begin{array}{cc}
H_{m+n-s}\left(S^{m} X\right) & \stackrel{h_{*}}{\longrightarrow} H_{m+n-s}\left(S^{n} Z\right) \\
\phi^{\prime} \downarrow & \downarrow \psi^{\prime} \\
H^{m+s}\left(S^{m} Y\right) \stackrel{\leftarrow}{\longleftarrow} H^{n+s}\left(S^{n} Y\right)
\end{array}
$$

Both $\sigma$ and $\psi^{\prime}$ are isomorphisms. If $\phi$, and hence $\phi^{\prime}$, is an isomorphism, then $h_{*}$ is an isomorphism. This proves (2.2).

3. Axial maps. We suppose that $P^{a}$ has its usual cell-structure, and denote its basepoint by $e$. When $r<q$ we regard $P^{r}$, in the usual way, as a subspace of $P^{q}$, and denote by $P^{q} / P^{r}$ the space obtained by collapsing $P^{r}$ to a point. This is a sphere, for example, if $r=q-1$. We say ${ }^{4}$ that $P^{q} / P^{r}$ is $S$-reducible if its mod 2 homology is $S$-spherical in dimension $q$. Let $\phi(m)$, where $m \geqq 0$, denote the number of values of $s$ in the range $0<s \leqq m$ which are congruent to $0,1,2$ or $4 \bmod 8$. The work of Adams [1] has determined the number which appears in (2.7) of [7], and so the necessary and sufficient condition for $S$ reducibility is that

$$
q+1 \equiv 0 \bmod 2^{\phi}, \text { where } \phi=\phi(q-r-1) .
$$

Consider the following problem of Hopf [6] (see also Behrend [4]). Let $1 \leqq j \leqq n, 1 \leqq k \leqq n$. By an axial map of $P^{i} \times P^{k}$ into $P^{n}$ we mean a map

$$
f: P^{i} \times P^{k} \rightarrow P^{n}
$$

such that if $x \in P^{i}$ and $y \in P^{k}$ then

$$
f(x, e)=x, \quad f(e, y)=y .
$$

When $n \geqq j+k$ the existence of an axial map can be proved by direct construction, as in $\$ 1$ of [6], or by use of obstruction theory. What is the least value of $n$, given $j$ and $k$, for which there exists such a map? We recall

Theorem (3.2) (Hopf). Suppose that there exists an axial map of $P^{i} \times P^{k}$ into $P^{n}$. Then the binomial coefficients $C_{n, s}$ all have the same parity for $n-j \leqq s \leqq k$.

The proof given in $\$ 2$ of [6] translates into cohomology theory as

4In integral homology, twice every class is spherical. Hence it follows that the present definition agrees with the one of $\$ 2$ of [7]. 
follows. Take coefficients in the ring of integers mod 2, and make the usual identification of the cohomology ring of a product space with the tensor product of the cohomology rings of the factors. An axial map of $P^{i} \times P^{k}$ into $P^{n}$ induces a ring homomorphism

$$
\theta: H^{*}\left(P^{n}\right) \rightarrow H^{*}\left(P^{j}\right) \otimes H^{*}\left(P^{k}\right)
$$

such that $\theta(\alpha)=\beta \otimes 1+1 \otimes \gamma$, where $\alpha, \beta, \gamma$ are 1-dimensional generators. Since $\alpha^{n+1}=0$ it follows by naturality that

$$
(\beta \otimes 1+1 \otimes \gamma)^{n+1}=0
$$

and so $\beta^{n+1-s} \otimes \gamma^{s}$ has coefficient zero in the binomial expansion when $n-j<s \leqq k$. Hence $C_{n+1, s}$ is even, and so $C_{n, s}$ has the same parity as $C_{n, 8-1}$. This proves (3.2).

Theorem (3.3). Suppose that there exists an axial map of $P^{i} \times P^{b}$ into $P^{n}$, and that $C_{n, s}$ is odd when $n-j \leqq s \leqq k$. Then $P^{i} / P^{j-t}$ is $\bmod 2$ $S$-dual to $P^{k} / P^{k-t}$, where $t=j+k-n+1$.

By cellular approximation we can suppose that the axial map

$$
f: P^{j} \times P^{k} \rightarrow P^{n}
$$

is cellular. Since $P^{n-1}$ contains the image of

$$
P^{j} \times P^{k-t} \cup P^{j-t} \times P^{k},
$$

for dimensional reasons, it follows that $f$ induces a map

$$
g:\left(P^{j} / P^{j-t}\right) \#\left(P^{t} / P^{k-t}\right) \rightarrow P^{n} / P^{n-1}=S^{n} .
$$

Since $C_{n, s}$ is odd we have

$$
\theta\left(\alpha^{n}\right)=\sum_{s=n-j}^{s=k} \beta^{n-8} \otimes \gamma^{s}
$$

and so dually

$$
\phi\left(\beta_{n-s}\right)=\gamma^{s} \quad(n-j \leqq s \leqq k),
$$

where $\beta_{n-s}$ denotes the dual of $\beta^{n-s}$ and

$$
\phi: H_{n-s}\left(P^{j}\right) \rightarrow H^{s}\left(P^{k}\right)
$$

is the slant operation determined by $f$ and $\alpha^{n}$. In other words $\phi$ is an isomorphism for $n-j \leqq s \leqq k$, and hence, by naturality, the same is true of the slant operation

$$
H_{n-s}\left(P^{i} / P^{j-t}\right) \rightarrow H^{s}\left(P^{k} / P^{k-t}\right)
$$

determined by $g$ and the fundamental class on $S^{n}$. Thus (3.3) follows from (2.2). 
In (6.1) of [2], Atiyah has determined the dual of $P^{k} / P^{k-t}$. By combining this result with (3.3) we obtain

Corollary (3.4). If the hypotheses of (3.3) are satisfied then $P^{j} / P^{j-t}$ is $\bmod 2 S$-related to $P^{l} / P^{l-t}$ where $l=j+m-n$ and $m+1$ is a large power of 2 .

We use (3.1) and (3.4) to prove

Theorem (3.5). Let $C_{n, j}$ be odd. Suppose that both $n+1$ and $j+1$ are odd multiples of $2^{r}$, for some $r \geqq 0$. Then there exists no axial map of $P^{j} \times P^{k}$ into $P^{n}$, where $k=n+q-j$ and $q$ is as in (1.1).

Suppose, to obtain a contradiction, that such an axial map exists. Then $\phi(t-1)=\phi(q)=r+1$, by calculation, and

$$
l+1 \equiv j-n \equiv 0 \bmod 2^{r+1} .
$$

Hence $P^{l} / P^{l-t}$ is $S$-reducible by (3.1) and so $P^{j} / P^{j-t}$ is $S$-reducible by (2.1) and (3.4), which contradicts (3.1).

Other results about axial maps can be deduced from (3.4) by direct use of the Adams $\psi$ operators (see Appendix).

4. Vector fields. By a $k$-field on $P^{j}$ of tangents to $P^{n}$ we mean a continuous function which assigns to each point of $P^{i}$ a set of $k$ linearly independent tangent vectors to $P^{n}$ at that point. The first part of the following theorem is due to Hopf [6], and the converse is a consequence of $(6.6)$ of [8].

Theorem (4.1). There exists an axial map of $P^{j} \times P^{k}$ into $P^{n}$ if $P^{j}$ admits a $k$-field of tangents to $P^{n}$. The converse is true if $j<2(n-k)$.

Let $G_{j}$ denote the reduced Grothendieck group of classes of real vector bundles over $P^{i}$. We recall (see [1]) that this group is cyclic of order a power of 2 . A generator is the class $\xi$ of the nontrivial linebundle. If $x \in G_{j}$ we can always choose $n$, where $n>j$, so that $x=(n+1) \xi$. Thus $x$ is represented as the restriction to $P^{j}$ of the tangent bundle to $P^{n}$. If $g$ is the geometric dimension of $x$, as defined in $\$ 1$ of [3], then $P^{j}$ admits a $k$-field of tangents to $P^{n}$ for $k=n-g$ but not for $k=n-g+1$. Moreover in many cases one can go on and use (4.1) to reduce the problem to one about axial maps. The deduction of (1.1) from (3.5) is a simple illustration of this procedure.

If $P^{j}$ is immersed in $(2 j-q)$-space then the normal $(j-q)$-plane bundle represents $-(j+1) \xi \in G_{j}$. Hence $P^{i}$ admits a $k$-field of tangents to $P^{n}$, where

${ }^{5}$ By using (1.4) of [3] it is sometimes possible, as in the immersion problem, to get round the restriction on the converse of (4.1). 


$$
n=m-(j+1), \quad k=n+q-j .
$$

Here $m+1$ is a large power of 2 , as before, which is divisible by the order of $G_{j}$. Now $C_{n, j}$ is odd, by the dyadic rule, since $j+1=2^{r}$. Also there exists an axial map of $P^{j} \times P^{k}$ into $P^{n}$, by (4.1). Thus the hypotheses of (3.5) are fulfilled and so (1.1) is obtained.

5. Reflexive maps. In [6] Hopf is primarily concerned not with axial maps but with "Definite Systeme ungerade Funktionen" which are equivalent, for present purposes, to reflexive maps, in the following sense. On any sphere let $T$ denote the antipodal transformation. We describe a map

$$
f: S^{j} \times S^{k} \rightarrow S^{n}
$$

as reflexive if, for all $x \in S^{i}$ and $y \in S^{k}$,

$$
f(T x, y)=T f(x, y)=f(x, T y) .
$$

We regard $S^{n}$ as a double covering of $P^{n}$ and regard $S^{j} \times S^{k}$ as a quadruple covering of $P^{j} \times P^{k}$. Since the inclusion maps

$$
P^{i} \rightarrow P^{n} \leftarrow P^{k}
$$

are covered by the inclusion maps

$$
S^{i} \rightarrow S^{n} \leftarrow S^{k}
$$

it follows that an axial map of $P^{j} \times P^{k}$ into $P^{n}$ can always be covered by a reflexive map of $S^{i} \times S^{k}$ into $S^{n}$. It can also be proved, using the methods of [8], that the existence of a reflexive map is a sufficient condition for the existence of an axial map.

THEOREM (5.1). If there exists an axial map of $P^{i} \times P^{k}$ into $P^{m}$ and an axial map of $P^{i} \times P^{k}$ into $P^{n}$ then there exists an axial map of $P^{i+j+1} \times P^{k}$ into $P^{m+n+1}$.

We lift the given axial maps to reflexive maps

$$
f: S^{i} \times S^{k} \rightarrow S^{m}, \quad g: S^{j} \times S^{k} \rightarrow S^{n} .
$$

An equivariant map $\zeta: S^{i+j+1} \rightarrow S^{m+n+1}$ is obtained by taking the join of equivariant maps

$$
\xi: S^{i} \rightarrow S^{m}, \quad \eta: S^{i} \rightarrow S^{n} .
$$

Let $x \in S^{i}, y \in S^{i}, z \in S^{k}, w \in S^{i+j+1}$. If $\xi(x)=f(x, z)$ and $\eta(y)=g(y, z)$ then we write $\zeta(w)=h(w, z)$, so as to define

$$
h: S^{i+j+1} \times S^{k} \rightarrow S^{m+n+1} .
$$


The map $h$ is reflexive, and induces an axial map of $P^{i+j+1} \times P^{k}$ into $P^{m+n+1}$, as required. Similarly ${ }^{6}$ one can prove

ThEOREM (5.2). If there exists $a k$-field on $P^{i}$ of tangents to $P^{m}$ and $a k$-field on $P^{j}$ of tangents to $P^{n}$ then there exists a $k$-field on $P^{i+j+1}$ of tangents to $P^{m+n+1}$.

6. Appendix. If one space is mod $2 S$-related to another, as in (3.4), then the Steenrod squares must correspond. Hence, as in (12.2) of [7], we obtain the preliminary

TheOREM (6.1). Let $C_{n, j}$ be odd. If there exists an axial map of $P^{i} \times P^{k}$ into $P^{n}$ then $n+1$ is a multiple of $c$, where $c$ denotes the least power of 2 which exceeds $j+k-n$.

The purpose of this Appendix is to show how the following result can be obtained by using the Adams $\psi$ operations in place of the Steenrod squares. When $j+k$ is much greater than $n$ the improvement over (6.1) is exponential.

THEOREM (6.2). Let $C_{n, j}$ be odd and let $k \neq \equiv$ mod 4 . If there exists an axial map of $P^{i} \times P^{k}$ into $P^{n}$ then $n+1$ is a multiple of $2^{\theta-1}$, where $\theta$ denotes the number of values of $s$ in the range $n-k \leqq s \leqq j$ which are congruent to $0,1,2$ or $4 \bmod 8$.

If $k \equiv 3 \bmod 4$ but otherwise the hypotheses are fulfilled one can deduce that $n+1$ is a multiple of $2^{\theta-2}$ by applying (6.2) to the pair $(j, k-1)$ rather than $(j, k)$. Also there are cases where a better result can be obtained by interchanging $j$ and $k$.

If $j+k \leqq n+3$ then (6.2) is a special case of (6.1). Suppose, therefore, that $j+k \geqq n+4$. Then $c \equiv 0 \bmod 8$, in (6.1) and so $n \equiv 7 \bmod 8$. Hence

$$
\begin{aligned}
& j-t+1 \equiv n-k \neq 0 \bmod 4, \\
& \iota-t+1 \equiv-(k+1) \neq \equiv 0 \bmod 4 .
\end{aligned}
$$

Let $\tilde{K}_{R}(X)$ denote the reduced Grothendieck group of classes of real vector bundles over a space $X$. By (7.4) of [1] the groups

$$
\tilde{K}_{R}\left(S^{m+1}\left(P^{i} / P^{j-t}\right)\right), \quad \tilde{K}_{R}\left(S^{n+1}\left(P^{l} / P^{l-t}\right)\right)
$$

are cyclic of order $2^{\theta}$, where $\theta$ is as in (6.2).

The method used by Serre in Chapter II of [9] can be applied to the Atiyah-Hirzebruch spectral sequence (see $\$ 6$ of [1]) connecting vector bundles to cohomology. When $\mathfrak{e}$ is the class of finite abelian

- These results are analogous to those obtained by Behrend [4] in the algebraic case. 
groups of odd order the following result is obtained. Let $X$ be a finite complex with trivial homology $\bmod 2$. Then $\widetilde{K}_{R}(X) \in \mathcal{e}$ since, by the universal coefficient theorem, $\mathfrak{e}$ contains the $\bmod 2$ and integral cohomology groups of $X$.

Under the hypotheses of (6.2) it follows from (3.4) above that there exists a map

$$
h: S^{m+1}\left(P^{i} / P^{j-t}\right) \rightarrow S^{n+1}\left(P^{l} / P^{l-t}\right)
$$

which induces an isomorphism of $\bmod 2$ homology. Take $X$ to be the mapping cylinder of $h$. Then $\tilde{K}_{R}(X) \in \mathcal{e}$, since $X$ has trivial mod 2 homology, and so the induced homomorphism

$$
h^{*}: \tilde{K}_{R}\left(S^{n+1}\left(P^{l} / P^{l-t}\right)\right) \rightarrow \widetilde{K}_{R}\left(S^{m+1}\left(P^{i} / P^{j-t}\right)\right)
$$

is a $\mathfrak{C}$-monomorphism. Both groups are cyclic of order $2^{\theta}$, as we have seen, and so $h^{*}$ is an isomorphism for algebraic reasons.

Consider the Adams $\psi_{R}^{q}$ operation, as defined in [1], where $q$ is odd. By (5.3) and (7.4) of [1] we have

$$
q^{(n+1) / 2} h^{*}=h^{*} \psi_{R}^{q}=\psi_{R}^{q} h^{*}=q^{(m+1) / 2} h^{*} .
$$

Since $h^{*}$ is an isomorphism this implies that $q^{(m+1) / 2} \equiv q^{(n+1) / 2}, \bmod 2^{\theta}$, and hence that $q^{(m-n) / 2} \equiv 1, \bmod 2^{\theta}$, since $q$ is odd. Take $q=3$. Then $\frac{1}{2}(m-n)$ is divisible by $2^{-\theta-2}$, by (8.1) of [1], and so $n+1$ is divisible by $2^{\theta-1}$, which proves $(6.2)$.

\section{REFERENCES}

1. J. F. Adams, Vector fields on spheres, Ann. of Math. 75 (1962), 603-632. 310.

2. M. F. Atiyah, Thom complexes, Proc. London Math. Soc. (3) 11 (1961), 291-

3. - Immersions and embeddings of manifolds, Topology 1 (1962), 125-132.

4. F. Behrend, Über Systeme reeler algebraischer Gleichungen, Compositio Math. 7 (1939), 1-19. 276.

5. M. Hirsch, Immersions of manifolds, Trans. Amer. Math. Soc. 93 (1959), 242-

6. H. Hopf, Ein topologischer Beitrag zur reelen Algebra, Comment. Math. Helv. 13 (1940-41), 219-239.

7. I. M. James, Spaces associated with Stiefel manifolds, Proc. London Math. Soc. (3) 9 (1959), 115-140.

8. - The space of bundle maps, Topology (to appear).

9. J.-P. Serre, Groupes d'homotopie et classes de groupes abeliens, Ann. of Math. 58 (1953), 258-294.

10. E. Spanier, Infinite symmetric products, function-spaces, and duality, Ann. of Math. 69 (1959), 142-198.

11. - Function spaces and duality, Ann. of Math. 70 (1959), 338-378.

MATHEMatical Institute, OXFord 